\title{
Lógica difusa para la toma de decisiones y la selección de personal
}

\section{Fuzzy Logic for Decision-Making and Personnel Selection}

\section{Lógica difusa para a tomada de decisões e a seleção de pessoal}

\section{Francisco Javier Ruvalcaba Coyaso Anäis Vermonden ${ }^{\star \star}$}

Fecha de recibido: 29 de enero de 2015

Fecha de aprobado: 30 de abril de 2015

Doi: dx.doi.org/10.12804/rev.univ.empresa.29.2015.10

Para citar este artículo: Ruvalcaba Coyaso, F. J., \& Vermonden, A. (2015). Lógica difusa para la toma de decisiones y la selección de personal. Universidad \& Empresa, 1729), 239-256. Doi: dx.doi.org/10.12804/ rev.univ.empresa.29.2015.10

\section{RESUMEN}

Este trabajo de investigación se enfoca en la lógica difusa. Identifica de qué manera sirve para la selección de personal y qué publicaciones relevantes existen acerca de su efectividad en el escenario empresarial. La revisión realizada se llevó a cabo a partir de una búsqueda en bases de datos especializadas. Se encuentra que la fuzzy logic puede ofrecer al proceso de selección de personal algo de certidumbre, en particular en la toma de decisiones que lo acompaña. Puede contribuir también en el proceso de identificación de la

* Doctor en Psicología por la Universidad de Colima (México), profesor investigador adscrito al Departamento de Psicología de la Universidad Autónoma de Aguascalientes (México); coordinador de la línea de investigación "Inserción y trayectorias laborales"; miembro del Sistema Nacional de Investigadores de CONACyT; asesor gubernamental y consultor privado. Correo electrónico: javiercoyaso@hotmail.com

** Maestra en Ciencias de la Tierra, Universidad Nacional Autónoma de México, México D.F. Estudiante de doctorado del Departamento de Geografía y Ambiente (Geography and Environment) de la Universidad de Southampton (Reino Unido). Correo electrónico: anaisvt@gmail.com 
persona más adecuada para realizar un conjunto de actividades, de acuerdo, además, con su perfil psicológico. Su uso ayuda, en efecto, a disminuir la ambigüedad y la subjetividad inherentes a la decisión en estos procesos. Esto, dado que los resultados de las pruebas psicométricas y de las entrevistas no son discrecionales, ellas se acompañan, usualmente, de múltiples criterios de asignación de valor.

Palabras clave: lógica difusa, modelos matemáticos, selección de personal, toma de decisiones.

\section{ABSTRACT}

This research focuses on fuzzy logic. It identifies how it can be used for the selection of personnel and the relevant publications on its effectiveness on the entrepreneurial context. The review was carried out from a search in specialized databases. It has been found that fuzzy logic can provide a degree of certainty to the personnel selection process, in particular in the decision making accompanying it. It can contribute to the identification process of the most appropriate person to carry out a series of activities according, in addition, to the respective psychological profile. In fact, its use helps to reduce ambiguity and subjectivity inherent in decisions in these processes, since the results of the psychometric tests and interviews are not discretional, they are usually accompanied by multiple value assignment criteria.

Keywords: Fuzzy logic, mathematical models, personnel selection, decision making.

\section{RESUMO}

Este trabalho de pesquisa foca-se na lógica difusa. Identifica de que maneira serve para a seleção de pessoal e que publicações relevantes existem acerca de sua efetividade no cenário empresarial. A revisão realizada se levou a cabo a partir de uma busca em bases de dados especializadas. Encontra-se que a Fuzzy Logic pode oferecer ao processo de seleção de pessoal algo de certeza, em particular, na tomada de decisões que o acompanha. Pode contribuir também no processo de identificação da pessoa mais adequada para realizar um conjunto de atividades, de acordo, além, com o seu perfil psicológico. Seu uso ajuda, em efeito, a diminuir a ambiguidade e a subjetividade inerentes à decisão nestes processos. Isto, dado que os resultados das provas psicométricas e das entrevistas não são discricionais, elas acompanham-se, usualmente, de múltiplos critérios de atribuição de valor.

Palavras-chave: lógica difusa, modelos matemáticos, seleção de pessoal, tomada de decisões. 


\section{INTRODUCCIÓN}

La presente investigación se enfoca en la lógica difusa. Identifica de qué manera sirve para la selección de personal y qué publicaciones relevantes existen acerca de su efectividad en el escenario empresarial.

El estudio se realizó con base en una revisión de literatura hecha gracias al uso de bases de datos especializadas. Se identifica que la fuzzy logic puede ofrecer al proceso de selección de personal algo de certidumbre, en particular, en lo relativo a la toma de decisiones que lo caracteriza.

Esta puede contribuir también en el proceso de identificación de la persona más adecuada para realizar un conjunto de actividades, de acuerdo incluso con su perfil psicológico (Murphy, 2000; Oostrom, Van der Linden, Born, \& Van der Molen, 2013; Verive \& McDaniel, 1996; Grand, Golubovich, Ryan, \& Schmitt, 2013). La utilidad puede obtenerse como señalan Formann (1992); Caki y Solmaz (2013); McDaniel (2009) y Jessop (2004), en la disminución de la ambigüedad y la subjetividad inherentes a la decisión en estos procesos; o bien en la disminución de accidentes teniendo la elección adecuada del personal (Jones \& Wuebker, 1988). Esto, dado que los resultados de las pruebas psicométricas y de las entrevistas no son discrecionales. Estas, por lo general, se acompañan de múltiples criterios de asignación de valor.

El texto tiene cinco partes. La primera se dedica a mostrar la transición de los procesos de selección personal hacia la consideración de modelos matemáticos que son una alternativa útil para sus propósitos. La segunda hace una mirada a la lógica difusa y la pone en contexto. La tercera describe los elementos metodológicos básicos considerados para hacer la revisión. La cuarta parte hace una síntesis de los resultados esenciales del trabajo. Finalmente, el quito incorpora algunos elementos de discusión y las conclusiones.

\section{LA TRANSICIÓN HACIA MODELOS MATEMÁTICOS}

La selección de personal en empresas y organizaciones ha estado constituida por procesos, decisiones $y$ herramientas que ofrecen información del candidato a través de pruebas psicométricas o entrevistas (McDaniel, 2009), para medir aptitudes (Zysberg \& Nevo, 2004), competencias (Shanteau, Weiss, Thomas, \& Pounds, 2002; Weinstein, 2012; Gutjahr, Katzensteiner, Reiter, Stummer, \& Denk, 2010), rasgos de personalidad (Rothstein \& Goffin, 2006; Vecchione,Alessandri, \& Barbarane- 
1li, 2012), valores y rasgos aspiracionales, incluso condiciones de igualdad para la empleabilidad (Gutman, 2009; Mael, 1998; Ralston, 1988). De acuerdo a Safarzadegan Gilan, Sebt, \& Shahhosseini (2012), la obtención de datos a través de las pruebas psicométricas no implica que la selección del candidato sea objetiva, pues estos continúan anclados de algún modo a la persona que los genera, de ahí la necesidad de contar con criterios definidos para generar incluso modelos consistentes (Özdaban \& Özkan, 2010; Storey Hooper, Galvin, Kilmer, \& Liebowitz, 1998; Bobko \& Roth, 2004).

Los procesos o flujos de procedimiento en la selección de personal son otro componente fundamental pues a través de estos y de su consistencia es posible tener una mayor confianza en la información ofrecida (Robertson \& Smith, 2001). Los procesos deben ser especificados para cada puesto dentro de la organización y es recomendable que se indique también el tipo de herramienta a utilizar en cada fase del proceso, incluso cuando se hacen a través de medios electrónicos (Neuman \& Nomoto, 1990).

La decisión es, usualmente, el tercer componente de la selección de personal. Es una parte esencial del proceso administrativo en materia de recursos humanos en empresas y organizaciones por el cual un conjunto de personas, con un determinado número y tipo de competencias o rasgos de personalidad, se somete a una evaluación para ocupar un puesto con determinadas exigencias o demandas. La decisión, dentro del proceso administrativo de selección de personal, ha incorporado tradicionalmente un importante nivel de subjetividad, incluso cuando se tienen referentes objetivos como los que pueden obtenerse a través de pruebas psicométricas u otras herramientas que ofrecen elementos de cuantificación como, por ejemplo, INSIGHT-C (Jabri, 1990).

Los procesos de selección de personal han conducido a las empresas y organizaciones a utilizar estrategias y procesos de selección que les eviten pérdidas, algunas incluso no pensadas como posibles, tal es el caso de personal con problemas de adicción o violencia (Martin \& Godsey, 1999; Slora, Joy, \& Terris, 1991). Los cambios en organizaciones, en los procesos de trabajo, las regulaciones y el marketing han influido en la manera en que se recluta y selecciona personal (Dursun \& Karsak, 2010; Chien, \& Chen, 2008; Diken, Özturk, Uzel, \& Yılmaz, 2011).

Esta necesidad por implementar estrategias altamente escrupulosas 
tiene un doble beneficio: por un lado, se contrata a la persona que mejor se ajusta a las necesidades de la empresa u organización $y$, por el otro, se deja de invertir tiempo y dinero en repetidos procesos de selección de personal.

En el primer caso hay un valor adicional a mediano y largo plazo, pues, como ha mostrado Blegen (1993), la satisfacción laboral está asociada a bajo desempeño laboral, compromiso en el trabajo, ausentismo y rotación. Así, por ejemplo, una persona que no logra ajuste en su puesto de trabajo presenta más problemas de salud que aquella que tiene un ajuste adecuado. Este ajuste es resultante, en gran medida, de una selección adecuada o inadecuada.

La decisión es la acción que define al candidato que habrá de ocupar un puesto de trabajo, por lo tanto, debe ser considerado un proceso que se encuentra insertado en el flujo de procedimiento para la selección de personal. Un proceso que, por su importancia, diversas organizaciones consideran cada vez más que debe ser desarrollado de manera razonada y formal y, en consecuencia, estiman que es relevante usar modelos matemáticos que permitan soportarlo de manera más adecuada. Estos modelos pueden sustentarse en múltiples aproximaciones, entre las disponibles se destaca la lógica difusa.

\section{LÓGICA DIFUSA (FUZZY LOGIC)}

La lógica difusa fue planteada por Zadeh en 1965. Constituye una generalización de la lógica clásica, que es determinista: "verdadero" o "falso". Sin embargo, la fuzzy logic, a diferencia de la lógica clásica, tiene fronteras imprecisas. De acuerdo a Zadeh, un conjunto difuso es una clase de objetos con un contínuum que muestra su grado de membresía de ese conjunto. En efecto, cada miembro del conjunto está caracterizado por una función de membresía, la cual va desde cero hasta a uno.

La lógica difusa, al ser una forma de lógica multivaluada, puede manejar el razonamiento aproximado. Por esta razón, las variables lingüísticas se utilizan en la definición de conjuntos. Así, por ejemplo, una variable lingüística como podría ser la edad, puede tener valores tales como el de joven y viejo. El valor joven permite categorizar a los elementos del universo de edad con mayor detalle al darle valores de que varían en pertenencia dentro del conjunto (Zadeh, 1965).

Otra ventaja importante es la transición de pertenencia de un conjunto 
a otro. Retomando el ejemplo de la variable de edad, consideremos dos conjuntos, uno $A$, jóvenes y otro $B$, viejos. Cada uno de estos tendrá una función que define su membresía del grupo y que será definida como $\mu_{\text {edad }}(x)$, en la cual $x$ representa el grado de membresía del conjunto. Por tanto, entre más cerca de uno (1) se encuentre el valor de $x$, mayor será la membresía de dicho conjunto.

Por lo anterior, ciertos valores del universo de la variable edad pueden pertenecer a ambos conjuntos. Matemáticamente esto se puede expresar de la siguiente manera:

$\mu_{\text {fowen }}(x)\left\{1-\frac{1}{(e d a d(x)-50)} 0^{20}\right.$

: edad $(x) \leq 30$

$: 30 \leq \operatorname{edad}(x) \leq 5 \mathrm{C}$ $50<\operatorname{edad}(x)$

$\mu_{v i \in j 0}(x)\left\{1-\frac{(30-e d a d(x))}{0^{20}}\right.$

$: \operatorname{edad}(x)<30$

: $30 \leq \operatorname{edad}(x) \leq 5 C$ $\operatorname{edad}(x) \geq 50$

En el conjunto de joven, aquí definido, alguien de 30 años es considerado joven, por lo que un individuo cuya edad se esté aproximando a los 50 va perdiendo pertenencia al conjunto de joven y, en cambio, ganando pertenencia al conjunto de viejos.

Li (2014), Alliger, Feinzig \& Janak (1993), Celik, Kandakoglu y Er (2009) y Dağdeviren (2010), comparten la idea de utilizar los conjuntos difusos (fuzzy sets) en escenarios de recur- sos humanos, ya que la utilización de instrumentos de medida como pruebas psicométricas, cuestionarios, guías de entrevista, entre otros, no siempre van acompañados de criterios definidos para las puntuaciones obtenidas en dichas pruebas.

Una situación sería tener baterías para la selección de personal y otra (complementaria) serían las baterías, además un modelo de asignación de valores obtenidos en dichas pruebas.

Siguiendo este orden de ideas, es posible asignar valores a cada una de las categorías a evaluar dentro del proceso de selección de personal, particularmente en la decisión (ver tabla 1). Es importante señalar que, considerando los planteamientos de Zadeh (1965), los valores asignados correspondientes a un cierto grado de pertenencia seguirían un contínuum, de manera que la especificidad en la asignación de valores depende de los objetivos que tienen en la selección.

Tabla 1. Valores asignados a la categoría, por ejemplo, de "coeficiente intelectual (CI)" en un trabajador

\begin{tabular}{|c|c|}
\hline CI & Grado de pertenencia \\
\hline 0 & 0 \\
\hline 50 & 0,05 \\
\hline 80 & 0,20 \\
\hline 100 & 0,5 \\
\hline 120 & 0,75 \\
\hline 140 & 1 \\
\hline
\end{tabular}

Fuente: Elaboración propia. 
Las funciones que definen la unión y la intersección de conjuntos difusos pueden generalizarse, a condición de que cumplan ciertas restricciones. Cumplen las condiciones de las funciones conorma triangular (t-conorma) que es la unión y norma triangular (t-norma), conocida también como la intersección. Los operadores principales que cumplen con las condiciones para ser t-conormas son el operador máximo y la suma algebraica $(\mathrm{O})$ y los operadores que cumplen para ser t-norma son el operador mínimo y el producto algebraico $(\mathrm{Y})$.

Los conjuntos y operadores difusos son sujetos y verbos de la lógica difusa. Al formular las reglas se usan enunciados condicionales de tipo "Si... entonces" que competen a la lógica difusa. La parte "Si" es el antecedente de la regla y la "Entonces", el consecuente o la conclusión.

Cada regla define una superficie de implicación. Para cada posible valor del antecedente se obtiene el conjunto difuso, realizando la implicación y la superposición de todos estos conjuntos difusos para formar la superficie de implicación de esa regla.

Todas las reglas que tienen el mismo consecuente (eligiendo un operador para realizar la implicación), definirán la misma superficie de implica- ción, pues esta superficie se construye para todos los valores que pueda tomar el antecedente (Pérez Pueyo, 2005). De la superficie de implicación se obtiene un valor concreto para cada regla, con la intersección de la superficie de implicación con el plano vertical que pasa por el valor del antecedente.

Hay dos formas de construir los sistemas de inferencia difusa, una se denomina Mamdani (Mamdani \& Assilian, 1975), en donde las salidas también son conjuntos difusos, la otra se llama Sugeno (1977), en donde las salidas son funciones lineales o constantes. El sistema de Mamdami es utilizado cuando se quiere imitar de manera más natural la inferencia humana.

El último paso en un sistema de inferencia difuso es la 'desfuzzificación'. Es un método por el cual se obtiene un valor de salida del conjunto difuso de salida, que es la agregación de todas las reglas. Los métodos más utilizados son:

- Método del máximo: Se elige como el valor de salida aquel para el cual la función característica del conjunto difuso es máximo. No es un método óptimo, pues el valor puede ser alcanzado por varias salidas.

- Método del centroide: Utiliza como salida el centro de gravedad 
de la función característica de salida. Con este método se obtiene una salida única.

- Método de la altura: Primero se calculan los centros de gravedad para cada regla del conjunto difuso de salida y después la media ponderada.

\section{METODOLOGÍA}

Los principales elementos del proceso de revisión de publicaciones se materializan en la tabla 2 . La revisión se llevó a cabo usando, en particular, las plataformas especializadas de Elsevier, Springer y Ebsco.

Las palabras clave utilizadas para la búsqueda fueron tres:

- "Personnel selection"

- "Fuzzy logic"

- "Fuzzy sets"

Esto se realizó haciendo que el sistema considerara la restricción de que aparecieran estos descriptores en tres sitios básicos: 1) título, 2) resumen (abstract) y 3) conceptos clave.

Luego de la revisión de los artículos que fue posible identificar en cada una de las bases de datos, se llevó a cabo una selección de artículos a partir de dos criterios: 1) que en el proceso ilustrado se hiciera referencia a la lógica difusa de Zadeh (1965) y 2) que estuviera basado el trabajo en una aplicación en el ámbito del capital humano, en particular en el de la selección de personal.

De los artículos encontrados y revisados se hizo una selección rigurosa para decantar los verdaderamente más relevantes para su presentación. Estos se relacionan en tabla 2. Ellos, en efecto, por una parte hacen referencia a la lógica difusa de manera central y, por la otra, consideran aplicaciones de la lógica difusa, específicamente, al proceso de selección de personal. Algunos de los artículos que aparecen en la búsqueda se refieren al analytic hierarchy process (Saaty, 1977), sin embargo, no es objeto del presente artículo explicarlo.

\section{RESULTADOS}

Como se ha indicado, en la tabla 2 se integran las publicaciones que, por su objetivo y contenido, recuperan de algún modo la lógica difusa original de Zadeh y cuya aplicación está relacionada con la selección de personal. Si bien el objetivo del presente texto no es discutir sobre los resultados obtenidos (en tanto efectividad de la aplicación al problema determinado), sí es posible referir de forma general la manera en que fue aplicada la lógica difusa y qué resultados y conclusiones ofrecen los autores de los documentos. 
Tabla 2. Publicaciones verdaderamente relevantes — decantadas a través del presente estudio - para el objetivo de la búsqueda

(Considerando de manera directa la lógica difusa de Zadeh [1965])

\begin{tabular}{|l|l|}
\hline \multicolumn{1}{|c|}{ Autor(es) y años } & \multicolumn{1}{c|}{ Artículo o publicación } \\
\hline Alliger et al. (1993) & "Fuzzy sets and personnel selection: discussion and an application". \\
\hline Dursun \& Karsak (2010) & "A fuzzy MCDM approach for personnel selection". \\
\hline $\begin{array}{l}\text { Canós, Casasús, Crespo, } \\
\text { Lara \& Pérez (2011) }\end{array}$ & "Personnel selection based on fuzzy methods". \\
\hline De Korvin \& Kleyle (2000) & $\begin{array}{l}\text { "A fuzzy set approach to resource procurement for multi-phase } \\
\text { projects subject to flexible budgetary constraints". }\end{array}$ \\
\hline Baležentis et al. (2012a) & $\begin{array}{l}\text { "MULTIMOORA-FG: A multi-objective decision making method } \\
\text { for linguistic reasoning with an application to personnel selection". }\end{array}$ \\
\hline Baležentis et al. (2012b) & $\begin{array}{l}\text { "Personnel selection based on computing with words and fuzzy } \\
\text { MULTIMOORA". }\end{array}$ \\
\hline Özdaban, \& Özkan (2010) & $\begin{array}{l}\text { "A fuzzy method on determining of job and personnel selection } \\
\text { results, and matching them with suggested model". }\end{array}$ \\
\hline $\begin{array}{l}\text { Kelemenis, \& Askounis } \\
\text { Özdaban, \& Özkan (2011). }\end{array}$ & $\begin{array}{l}\text { "A case study on evaluating personnel and jobs jointly with fuzzy } \\
\text { distances" }\end{array}$ \\
\hline
\end{tabular}

Fuente: Elaboración propia.

Alliger et al. (1993) es la primera publicación censada en que se hace referencia al Modelo de Zadeh. Estos autores presentan una propuesta concreta para la incorporación de la lógica difusa en el proceso final de la selección de personal, es decir, en la toma de decisión, otorgando valores específicos a los rasgos evaluados. Zadeh (1965) demostró que las leyes de Morgan se cumplen para los fuzzy sets, así que Alliger et al. (1993) generan un modelo de asignación de valor a los criterios de selección. Esto, utilizando las pro- piedades de unión e intersección que propone Yager (1981).

Afshari, Yusuff y Derayatifar (2013) y Dursun y Karsak (2010), por su parte, llevaron a cabo una propuesta en la cual integran la lógica difusa de Zadeh (1965), pero lo hacen incluyéndolo en un modelo multicriterio en el que consideran también el Modelo de representación lingüística 2-tupla (2-tuple linguistic representation model) y la technique for order preference by similarity to ideal solution (TOPSIs, por sus siglas 
en inglés), una técnica para la toma de decisiones multicriterio, ampliamente aceptada y utilizada (Behzadian, Otaghsara, Yazdani, \& Ignatius, 2012).

El objetivo de Afshari et al. (2013) y Dursun \& Karsak (2010) es proveer un modelo que permita la decisión y limite la ambigüedad en la asignación de valores lingüísticos: palabras implicadas en la decisión, como habilidad organizativa, creatividad, personalidad o liderazgo están significativamente asociadas a la ambigüedad, incluso cuando se miden con las pruebas psicométricas.

El punto de partida para Canós et al. (2011) es generar un modelo de elección que permita escoger al "candidato ideal" a través del proceso de selección de personal. Estos autores consideran que los procesos de selección de personal inician a partir de la revisión de las competencias del candidato. Las competencias consideradas son conocimiento, habilidades, actitudes y comportamientos, que muestra la manera en que el candidato se desempeñaría en ciertas tareas. Estas competencias deben poder observarse y medirse y, una vez verificado, puede entonces recibir una asignación de valor para el modelo matemático.

Las competencias del candidato son evaluadas usando intervalos de distancia, por lo cual, Canós et al. (2011) incorporan sistemas de medida de intervalo como hamming distance o matching level index (Dubois \& Prade, 2000). Estos intervalos de distancia se dan entre el candidato y "el ideal" (el ocupante perfecto para el puesto). Entre menor es la distancia entre el candidato y el ideal, mucho mejor.

Los intervalos de valor como $\phi-$ fuzzy permiten también un mejor manejo de los valores que representan las competencias. El best fit referido por Canós et al. (2011) es la búsqueda de una medida adecuada para determinar la distancia entre el candidato y el ideal y, aunque refiere la utilización de otras medidas de distancia como la Euclideana o la de Tchebichev, opta por las referidas basadas en la lógica difusa.

De Korvin y Kleyle (2000), por su parte, elaboran una propuesta de algoritmo que permite identificar la distancia entre las habilidades requeridas en un proyecto multifase y las habilidades que un candidato posee para ir cumpliendo con estos requerimientos. Es de algún modo un planteamiento similar al de Canós et al. (2011), pues estos autores tratan de generar un estándar objetivo de la diferencia (un criterio de ajuste). En el algoritmo propuesto por De Korvin y Kleyle (2000) se 
considera cada fase del proyecto con un número finito de habilidades requeridas, en el cual la membresía del conjunto representa el grado en que estas habilidades son requeridas para completar exitosamente la fase correspondiente.

Baležentis, Baležentis y Brauers (2012a, 2012b) incorporan también la lógica difusa en dos publicaciones sobre MULTIMOORA-FG, un método múltiple-objetivo, que los autores consideran una extensión del método llamado multi-objective optimization by ratio analysis (MOORA). Acentúan la importancia de contar con un referente que no sea vago, impreciso y ambiguo para la toma de la decisión. Recuperan la lógica difusa y la incorporan en los modelos MODM para toma de decisiones.

\section{DISCUSIÓN Y CONCLUSIONES}

$\mathrm{Al}$ inicio del texto se plantearon dos objetivos: por un lado, presentar la lógica difusa como herramienta para la modelación para la selección de personal y, por otro, revisar la efectividad de la lógica difusa en el proceso de selección de personal en el escenario organizacional o empresarial.

En el texto original de Zadeh (1965) no solo se presenta el planteamiento sobre la ambigüedad existente en el uso de palabras y términos, sino también en las limitaciones de la asignación binaria $(0,1)$ para determinar la membresía de un grupo. Para este autor, esta pertenencia a conjuntos no es absoluta, sino existente en un contínuum que va desde cero hasta uno. En su planteamiento, mostró que las operaciones algebraicas eran contenidas en el modelo y que las leyes de Morgan podían ser también explicadas desde la lógica difusa, a saber: unión, complementación e intersección.

Las aplicaciones expuestas en los artículos de Alliger et al. (1993), Dursun y Karsak (2010) y Canós et al. (2011), Licata (2006), Zadeh (2002), Kabak, Burmaoglu y Kazancoglu, (2012) y Zadeh (2009), muestran de manera clara una función en el proceso de selección de personal con la asignación concreta de valores a cada habilidad deseada en el candidato. Esto permite tomar una decisión basada exclusivamente en resultados cuantificables.

La eliminación de vaguedad, imprecisión y ambigüedad en el proceso de selección del personal es fundamental para estos autores, quienes ven útil y factible el modelo, incluso para situarse al ritmo de las exigencias contemporáneas de eficiencia. La utilización de consistentes herramientas para obtener información, 
como test o entrevistas bien estructuradas, no es muy útil si no se cuenta con un sistema que transforme esos datos en valores para procesar matemáticamente la decisión, ya sea dentro de la empresa o a través de un centro de evaluación externo (Adler, 1987; Thornton \& Gibbons, 2009).

Canós et al. (2011) y De Korvin y Kleyle (2000) utilizan el modelo asignando valores a cada habilidad requerida y otros a la habilidad del candidato. Esto constituye de algún modo la creación de grupos de membresía tal y como es planteado por Zadeh (1965). La menor distancia entre el ideal para el puesto y el candidato define la decisión. Los argumentos de estos autores, cercanos a una versión de distancia muestra, dan cuenta de una funcionalidad muy adecuada incluso para identificar las carencias y los campos de desarrollo para los candidatos, si es que son incorporados a la empresa u organización.

Las publicaciones en que se da cuenta y uso de la lógica difusa de Zadeh (1965) es importante en número, aunque al situar a esta en el contexto organizacional se reduce significativamente. En estas publicaciones pueden encontrarse propuestas e investigaciones como las de Jereb, Rajkovic, y Rajkovic (2005) y Díaz Mora, Piña, Ríos y Serafín (2009) en que se utilizan tanto el modelo de conjuntos difusos como el Proceso Analítico Jerárquico, indicado en Saaty, Peniwati y Shang (2007), Lin (2010) y en Saaty y Vargas (2012), con un caso particular: la selección de empresas contratistas, o bien en el planteamiento de Özdaban y Özkan (2011), quienes utilizan la lógica difusa en la evaluación del personal que ya se encuentra laborando en la empresa. Esta es una versión muy similar a las de Canós et al. (2011) y De Korvin y Kleyle (2000) acerca de la definición de la distancia entre lo que el trabajador está produciendo y lo que debería producir.

Aunque, como se observa, es bastante reducido el número de publicaciones en que se establece claramente la utilización de la lógica difusa de Zadeh (1965), puede considerarse, por otro lado, que su existencia en una situación tan concreta como la de toma de decisión al interior del proceso de selección de personal, da cuenta de que la lógica difusa es vista ya como una herramienta factible y útil, no solo para el proceso referido, sino para otros que se presentan en el escenario empresarial. Esto a partir de trabajos que, aunque pocos, son rigurosos, formalmente correctos, pertinentes y útiles. A manera de resumen, Saad, Ahmad, Abu y Jusoh, (2013) hacen un análisis breve pero claro sobre algunas 
de las técnicas derivadas de la lógica difusa que puede orientar y dar pauta para el uso de esta innovadora forma de selección de personal.

Finalmente, se observa en la lógica difusa una flexibilidad suficiente como para ajustarse a otros modelos y estrategias matemáticas como el Proceso Analítico Jerárquico, así como a procesos más amplios, como el de los diversos métodos para la toma de decisiones, tal y como se hace en el trabajo de Güngör, Serhadlıoğlu y Kesen (2009).

\section{REFERENCIAS}

Adler, S. (1987). Toward the more efficient use of assessment center technology in personnel selection. Journal of Business and Psychology, 2(1), p. 74-93.

Afshari, A. R., Yusuff, R. M., \& Derayatifar, A. R. (2013). Linguistic extension of fuzzy integral for group personnel selection problem. Arabian Journal for Science and Engineering, 38(10), p. 2901-2910.

Alliger, G. M., Feinzig, S. L., \& Janak, E. (1993). Fuzzy sets and personnel selection: Discussion and an application. Journal of Occupational and Organizational Psychology, 66, 163-169.

Baležentis, A., Baležentis, T., \& Brauers, W. K. M.(2012a). MUL-
TIMOORA-FG: A multi-objetive decisión making method for linguistic reasoning with an application to personnel selection.Informatica, 23(2), 173-190.

Baležentis, A., Baležentis, T., \& Brauers, W. K. M. (2012b). Personnel selection based on computing with words and fuzzy MULTIMOORA. Expert Systems with applications,39,7961-7967.

Behzadian, M., Otaghsara, K., Yazdani, M., Ignatius, J. (2012). A state-of the-art survey of TOPSIS applications. Expert Systems with Applications, 39, 13051-13069. Blegen, M. A. (1993). Nurses' job satisfaction: A meta-analysis of related variables. Nursing Research, 42(1), 36-41.

Bobko, P., \& Roth, P. (2004). Personnel selection with top-score-referenced banding: on the inappropriateness of current procedures. International Journal of Selection and Assessment, 12(4), 291-298.

Caki, N., \& Solmaz, B. (2013). The effects of facial beauty in personnel selection: A field work in retail sector. Procedia. Social and Behavioral Sciences, 84, 1203-1206.

Canós, L., Casasús, T., Crespo, E., Lara, T., \& Pérez, J. (2011). Personnel selection based on fuzzy methods. Revista de Matemática: Teoría y Aplicaciones, 18(1), 177-192. 
Celik, M., Kandakoglu, A., \& Er, D. (2009). Structuring fuzzy integrated multi-stages evaluation model on academic personnel recruitment in MET institutions. Expert Systems with Applications, 36, 6918-6927.

Chien, C.-F., \& Chen, L.-F. (2008). Data mining to improve personnel selection and enhance human capital: A case study in high-technology industry. Expert Systems with Applications, 34(1), 280-290.

Dağdeviren, M. (2010). A hybrid multi-criteria decisión-making model for personnel selection in manufacturing systems. Journal of International Manufacturing, 21, 451-460.

De Korvin, A., \& Kleyle, R. (2000). A fuzzy set approach to resource procurement for multi-phase projects subject to flexible budgetary constraints. Journal of Intelligent and Fuzzy Systems, 9, 225-233.

Díaz Mora, R., Piña, J., Ríos, D., Serafín, M. (2009). Uso de AHP y conjuntos difusos para mejorar la toma de decisiones. Caso: selección de empresas contratistas de construcción en la Administración Pública Venezolana (Ponencia presentada a la $7^{\mathrm{a}}$ Latin American and Caribbean Conference for Engineering and Technology, San Cristóbal, Venezuela).

Diken, E. H., Özturk, G., Uzel, N., \& Y1lmaz, M. (2011). Determi- ning the opinions of the prospective (candidate) teachers about public personnel selection exam (KPSS). Procedia Social and Behavioral Sciences, 15, 3571-3575.

Dubois, D., \& Prade, H. M. (Eds.) (2000). Fundamentals of fuzzy sets. Boston: Kluwer Academic. Dursun, M., \& Karsak, E. E. (2010). A fuzzy MCDM approach for personnel selection. Expert Systems with Applications, 37(6), 4324-4330.

Formann, A. K. (1992). Academic personnel selection: Description and prognosis of the decisions made by the committee for the selection of candidates for a full professorship. Scientometrics, 25(3), 401-414.

Grand, J. A., Golubovich, J., Ryan, A. M., \& Schmitt, N. (2013). The detection and influence of problematic item content in ability tests: An examination of sensitivity review practices for personnel selection test development. OrganizationalBehaviorand Human Decision Processes, 121(2), 158-173.

Gutjahr, W. J., Katzensteiner, S., Reiter, P., Stummer, C., \& Denk, M. (2010). Multi-objetive decision analysis for competence-oriented project portfolio selection. European Journal of Operational Research, 205, 670-679.

Gutman, A. (2009). Major EEO issues relating to personnel selec- 
tion decisions. Human Resource Management Review, 19(3), 232-250.

Güngör, Z., Serhadlıoğlu, G., \& Kesen, S. E. (2009). A fuzzy AHP approach to personnel selection problem. Applied Soft Computing, 9(2), 641-646.

Jabri, M. M. (1990). Personnel selection using INSIGHT-C: An application based on the analytic hierarchy process. Journal of Business and Psychology, 5(2), 281-285.

Jereb, E., Rajkovic, U., \& Rajkovic, V. (2005). A hierarchical multi-attribute system approach to personnel selection. International Journal of Selection and Assessment, 13(3), 198-205.

Jessop, A. (2004). Minimally biased weight determination in personnel selection. European Journal of Operational Research, 153(2), 433-444

Jones, J. W., \& Wuebker, L. J. (1988). Accident prevention through personnel selection. Journal of Business and Psychology, 3(2), 187198.

Kabak, M., Burmaoglu, S., \& Kazancoglu, Y. (2012). A fuzzy hibrid MCDM approach for professional selection. Expert Systems with Applications, 39, 3516-3525.

Kelemenis, A., Askounis, D. (2010). A new TOPSIS-based multi- criteria approach to personnel selection. Expert Systems with Applications, 37(7), 4999-5008.

Li, D. F. (2014). Multiattribute Decision-Making Methods with Intuitionistic Fuzzy Numbers. Studies in Fuzziness and Soft Computing, 308, 225-250.

Licata, I. (2006). General system theory, like-quantum semantics and fuzzy sets. En G. Minati, \& M. Abram (Eds.). System of Emergence, Research and Development. Nueva York: Springer (pp. 724-734).

Lin, H. (2010). Personnel selection using analytic network process and fuzzy data envelopment analysis approaches. Computers \& Industrial Engineering, 59, 937 944.

Mael, F. A. (1998). Privacy and personnel selection: reciprocal rightsandresponsibilities.Employee Responsibilities and Rights Journal, 11(3), 187-214.

Mamdani,E.H.,\&Assilian,S.(1975). An experiment in linguistic synthesis with a fuzzy logic controller. International Journal of Man-MachineStudies, 7(1), 1-13.

Martin, S. L., \& Godsey, C. (1999). Assessing the validity of a theoretically-based substance abuse scale for personnel selection. Journal of Business and Psychology, 13(3), 323-337.

McDaniel, M. A. (2009). Gerrymandering in personnel selection: 
A review of practice. Human Resource Management Review, 19(3), 263-270.

Murphy, K. R. (2000). Impact of assessments of validity generalization and situational specificity on the science and practice of personnel selection. International Journal of Selection and Assessment, 8(4), 194-206.

Neuman, G. A., \& Nomoto, J. T. (1990). Personnel selection tests for computer professionals and support technicians. Journal of Business and Psychology, 5(2), 165-177.

Oostrom, J. K., van der Linden, D., Born, M. P., \& van der Molen, H. T. (2013). New technology in personnel selection: How recruiter characteristics affect the adoption of new selection technology. Computers in Human Behavior, 29(6), 2404-2415.

Özdaban, I., Özkan, C. (2010). A fuzzy method on determining of job and personnel evaluation results, and matching them with suggested model. International Journal of Industrial Engineering, 17(4), 334-340.

Özdaban, I., \& Özkan, C. (2011). A case study on evaluating personnel and jobs jointly with fuzzy distances. International Journal of Industrial Engineering, 18(4), 169-179.

Pérez Pueyo, R. (2005). Procesado y optimización de espectros Ra- man mediante técnicas de lógica difusa: Aplicación a la identificación de materiales pictóricos (Tesis inédita, Departament de Teoria del Senyal i Comunicacions, Universitat Politècnica de Catalunya, Barcelona, España).

Ralston, S. M. (1988). The effect of applicant race upon personnel selection decisions: A review with recommendations. Employee Responsibilities and Rights Journal, 1(3), 215-226.

Robertson, I. T., \& Smith, M. (2001). Personnel selection. Journal of Occupational and Organizational Psychology, 74, 441-472.

Rothstein, M. G., \& Goffin, R. D. (2006). The use of personality measures in personnel selection: What does current research support? Human Resource Management Review, 16, 155-180.

Rothstein, M. G., \& Goffin, R. D. (2006). The use of personality measures in personnel selection: What does current research support?. Human Resource Management Review, 16(2), 155-180. Safarzadegan Gilan, S., Sebt, M. H., \& Shahhosseini, V. (2012). Computing with words for hierarchical competency based selection of personnel in construction companies. Applied Soft Computing, 12(2), 860-871.

Saad, R. M., Ahmad, M. Z., Abu, M. S., \& Jusoh, M. S. (2013). Some 
Fuzzy Techniques for Staff Selection Process: A Survey. Proceedings of the 20th National Symposium on Mathematical Sciences, 462-469.

Saaty, T. (1977). A scaling method for priorities in hierarchical structures. Journal of Mathematical Psychology, 15(3), 234-281. Shanteau, J., Weiss, D. J., Thomas, R. P., \& Pounds, J. C. (2002). Performance-based assessment of expertise: how to decide if a person is expert or not. European Journal of Operational Research, 136, 253-263.

Slora, K. B., Joy, D. S., \& Terris, W. (1991). Personnel selection to control employee violence. Journal of Business and Psychology, 5(3), 417-426.

Storey Hooper, R., Galvin, T. P., Kilmer, R. A., \& Liebowitz, J. (1998). Use of an expert system in a personnel selection process. Expert Systems with Applications, 14(4), 425-432.

Saaty, T. L., \& Vargas, L. G. (2012). Models, methods, concepts \& applications of the Analytic Hierarchy Process. Nueva York: Springer Science+Business Media.

Saaty, T. L., Peniwati, K., \& Shang, J. S. (2007). The analytic hierarchy process and human resource allocation: Half the history. Mathematical and Computer Modelling, 46, 1041-1053.
Sugeno, M. (1977). Fuzzy measures and fuzzy integrals: A survey. En M. M. Gupta, G. N. Saridis \& B. R. Gaines (Eds.), Fuzzy automata and decision processes (p. 89-102). Nueva York: NorthHolland.

Thornton, G. C., \& Gibbons, A. M. (2009). Validity of assessment centers for personnel selection. Human Resource Management Review, 19(3), 169-187.

Vecchione, M., Alessandri, G., \& Barbaranelli, C. (2012). The five factor model in personnel selection: Measurement equivalence between applicant and non-applicant groups. Personality and Individual Differences, 52(4), 503508.

Verive, J. M., \& McDaniel, M. A. (1996). Short-term memory tests in personnel selection: Low adverse impact and high validity. Intelligence, 23(1), 15-32.

Weinstein, D. (2012). The psychology of behaviorally-focused re'sume's on applicant selection: Are your hiring managers really hiring the 'right' people for the 'right' jobs? Business Horizons, 55, 53-63.

Yager, R. R. (1981). Anew methodology for ordinal multiobjetive decisions based on fuzzy sets. Decision Sciences, 12, 589-600.

Zadeh, L. A. (1965). Fuzzy sets. Information and Control, 8, 338353. 
Zadeh, L. A. (2002). Toward a perception-based theory of probabilistic reasoning with imprecise probabilities. Journal of Statistical Planning and Inference, 105, 233-264.

Zadeh. L. A. (2009). Toward extended fuzzy logic. Afirst step. Fuzzy SetsandSystems, 160,3175-3181.

Zhang, S., Liu, S. (2011). A GRAbased intuitionistic fuzzy multi- criteria group decision making method for personnel selection. Expert Systems with Applications, 38(9), 11401-11405.

Zysberg, L., \& Nevo, B. (2004). The Smarts That Counts?: Psychologists. Decision-Making in Personnel Selection. Journal of Business and Psychology, 19(1), 117124. 